\title{
The effect of chorda tympani section on ipsilateral and contralateral salivary secretion and taste in man
}

\author{
R GRANT,* S MILLER, $†$ D SIMPSON, $\ddagger$ P J LAMEY $\dagger$ I BONE§ \\ From the Neurology Unit, Department of Clinical Neurosciences, Western General Hospital,* Edinburgh; \\ Department of Oral Medicine and Pathology, Glasgow Dental Hospital and School, $\uparrow$ Glasgow; Ear, Nose and \\ Throat Department, Stobhill General Hospital, $\ddagger$ Glasgow; Department of Neurology, $\S$ Institute of Neurological \\ Sciences, Southern General Hospital, Glasgow, UK
}

SUMMARY Bilateral chorda tympani section is an accepted treatment for troublesome sialorrhoea. Nevertheless the effects of this operation have been poorly studied. Twenty patients were studied with unilateral chorda tympani section and a healthy contralateral ear. The effects on ipsilateral and contralateral stimulated submandibular and parotid flow and taste recognition and detection thresholds were measured. Stimulated submandibular flow accounted for only $27 \%$ of the total salivary flow. Chorda tympani section had no effect on submandibular flow in seven patients and only reduced submandibular flow by approximately $54 \%$ in the remaining 13 patients. No significant effect was observed on ipsilateral parotid flow rate. Electrogustometric taste detection thresholds were more than twice the accepted upper limit of normal on the lesioned side and taste recognition thresholds were also markedly abnormal. Chorda tympani section alone is a poor method of reducing stimulated salivary flow.

Chorda tympani section has been proposed as a treatment of drooling in bulbar motor neuron disease, following brain trauma and in mentally retarded patients. The chorda tympani nerve is thought to contain not only the special visceral afferent fibres of taste, but also the efferent parasympathetic secretomotor fibres to the submandibular and sublingual glands. If chorda tympani section is to be a successful method of treating drooling it should result in a significant reduction in the total salivary flow. However, little is known about the size of reduction in salivary flow rate or the severity of disruption of taste following chorda tympani section, as most studies have been performed on mentally retarded individuals ${ }^{1-3}$ in whom accurate measurements of salivary flow and taste are almost impossible.

To define more accurately the effects of chorda tympani section on salivary flow rate and taste, we measured stimulated salivary flow rates and taste detection and recognition thresholds in patients with

Address for reprint requests: Dr R Grant, Department of Clinical Neurosciences, Western General Hospital, Crewe Road, Edinburgh EH4 2XU, UK.

Received 28 October 1988 and in revised form 24 February 1989. Accepted 3 March 1989 operatively proven unilateral complete chorda tympani section.

The aims of this study were: firstly, to estimate the size of reduction in stimulated submandibular salivary flow rate; secondly, to assess whether the ipsilateral parotid flow rate was altered by chorda tympani section; thirdly, to determine the electrogustometric taste detection thresholds in patients with complete chorda tympani section and lastly, to establish whether all chemical taste was lost on the ipsilateral side of the tongue.

\section{Subjects and methods}

Twenty patients undergoing exploratory ear surgery were studied. The operations performed were mastoidectomy, stapedectomy or tympanoplasty. There were 11 females and nine males and ages ranged from 18-74 years (mean 43 years). Patients were included in the study if: $(a)$ there was no history of salivary or taste disturbance; $(b)$ the chorda tympani nerve was sectioned or found to be completely disrupted at the time of surgery; and $(c)$ the patient had a healthy contralateral ear.

Eleven of the patients had salivary flow rates measured within eight weeks of operation and nine had flow rates measured after eight weeks.

We decided to examine patients post-operatively only, and to compare taste and salivary flow rates on each side because 
it was not possible to predict pre-operatively which patients would require complete chorda tympani section for technical reasons at the time of operation, or which would have a totally disrupted chorda tympani due to local ear disease. There can also be significant variability in repeated measurements of salivary flow in healthy subjects but there is generally less interside variability.

\section{Salivary flow rates}

Parotid salivary flow was measured by locating the parotid duct, placing a Carlson Crittenden suction cup over the duct, stimulating salivary secretion using $0.5 \mathrm{ml}$ of $10 \%$ citric acid dropped onto the tongue and measuring the maximum flow in $\mathrm{ml} / \mathrm{min}$ over three consecutive minutes. The secretions from both parotid ducts were collected simultaneously.

Stimulated submandibular salivary flow rates were measured simultaneously after locating, dilating and cannulating the submandibular duct. Some patients required periductal lignocaine prior to cannulation. Submandibular secretion was stimulated with $0.5 \mathrm{ml}$ of $10 \%$ citric acid and secretions were collected with the patient in the "chin down" position to facilitate submandibular salivary flow.

\section{Electrogustometric taste detection thresholds (EGTDT)}

EGTDTs were measured using a previously standardised electrogustometer. ${ }^{4}$ The electrogustometer had a range from $1 \mu \mathrm{amp}$ to $999 \mu \mathrm{amps}$. Direct current is detected at a lower threshold than alternating current and anodal taste (acid, metallic) is more easily recognised than cathodal taste. ${ }^{4}$ For these reasons, the anodal electrode was used as the active electrode using direct current.

The active electrode was placed on the antero-lateral border of the tongue and the cathodal electrode was hand held by the patient to complete the circuit. The current was increased until the patient could detect an acidic or metallic taste. The current was then switched from the continuous mode to the pulsed mode and the settings were adjusted so that the current was on for five seconds and then off for five seconds automatically. When the current was on, a green LED would light up on the control panel, and when the current was off the light would switch off. The patient had to identify the onset of the current correctly in at least three out of four occasions. The current was gradually reduced until the taste detection threshold was reached, that is when the patient could no longer detect the onset of the current in at least three out of four occasions at one level of current.

\section{Chemical taste recognition and detection thresholds}

Chemical taste recognition and detection thresholds were measured using four standard solutions at three concentrations (sodium chloride $1 \cdot 5 \%, 7 \cdot 5 \%$ and $15 \%$; citric acid $1 \%$, $5 \%$ and $10 \%$; saccharin $1 \%, 10 \%$ and $40 \%$; quinine hydrochloride $0.075 \%, 0.5 \%$ and $1 \%$ ). Drops of solution were placed on one side of the tongue and the patient asked to detect or recognise the taste with the tongue held out. Three responses were recorded: $(a)$ correct recognition; $(b)$ detection of a taste but inability to recognise it; $(c)$ inability to detect any taste from the solution.

\section{Results}

\section{Salivary flow rates}

The mean salivary flow rates for each patient are shown in table 1 . The figure shows the mean, range and (x1) standard deviation for maximum salivary flow rate from $(a)$ the submandibular duct on the sectioned side and $(b)$ the submandibular duct on the normal side, $(c)$ the parotid duct on the sectioned side and $(d)$ the parotid duct on the normal side.

The stimulated submandibular flow rate was considerably less than the parotid flow rate and only contributed $27 \%$ of the total measured salivary flow.

Table 1 Individual results of submandibular and parotid stimulated salivary flow rates following unilateral chorda tympani section

\begin{tabular}{|c|c|c|c|c|c|}
\hline \multirow[b]{2}{*}{$\begin{array}{l}\text { Subject } \\
\text { No. }\end{array}$} & \multirow[b]{2}{*}{ Age (yr) } & \multicolumn{4}{|c|}{ Submandibular and parotid salivary flow rates } \\
\hline & & $\begin{array}{l}\text { Ipsilateral submandib } \\
(\mathrm{ml} / \mathrm{min})\end{array}$ & $\begin{array}{l}\text { Ipsilateral parotid } \\
\text { (ml/min) }\end{array}$ & $\begin{array}{l}\text { Contralateral submandib } \\
(\mathrm{ml} / \mathrm{min})\end{array}$ & $\begin{array}{l}\text { Contralateral parotid } \\
(\mathrm{ml} / \mathrm{min})\end{array}$ \\
\hline $\begin{array}{r}1 \\
2 \\
3 \\
4 \\
5 \\
6 \\
7 \\
8 \\
9 \\
10 \\
11 \\
12 \\
13 \\
14 \\
15 \\
16 \\
17 \\
18 \\
19 \\
20\end{array}$ & $\begin{array}{l}18 \\
19 \\
20 \\
28 \\
32 \\
34 \\
40 \\
40 \\
42 \\
44 \\
47 \\
49 \\
49 \\
50 \\
51 \\
52 \\
54 \\
60 \\
60 \\
74\end{array}$ & $\begin{array}{l}0.2 \\
0.2 \\
0.3 \\
0.2 \\
0.2 \\
0.1 \\
0.1 \\
0.2 \\
0.1 \\
0.4 \\
0.3 \\
0.2 \\
0.2 \\
0.1 \\
0.2 \\
0.1 \\
0.1 \\
0.2 \\
0.2 \\
0.1\end{array}$ & $\begin{array}{l}0.6 \\
0.5 \\
0.7 \\
0.8 \\
1.1 \\
1.0 \\
0.9 \\
0.9 \\
0.7 \\
0.5 \\
1.5 \\
0.6 \\
0.9 \\
0.5 \\
0.3 \\
0.5 \\
1.5 \\
1.3 \\
0.5 \\
1.6\end{array}$ & $\begin{array}{l}0.4 \\
0.4 \\
0.4 \\
0.3 \\
0.5 \\
0.3 \\
0.3 \\
0.2 \\
0.2 \\
0.2 \\
0.4 \\
0.2 \\
0.2 \\
0.3 \\
0.2 \\
0.3 \\
0.1 \\
0.2 \\
0.5 \\
0.3\end{array}$ & $\begin{array}{l}0.7 \\
0.6 \\
1.2 \\
0.4 \\
2.5 \\
0.9 \\
1.2 \\
0.8 \\
0.9 \\
0.7 \\
0.9 \\
0.5 \\
1.1 \\
0.4 \\
0.4 \\
0.5 \\
1.2 \\
1.4 \\
0.5 \\
1.4\end{array}$ \\
\hline
\end{tabular}



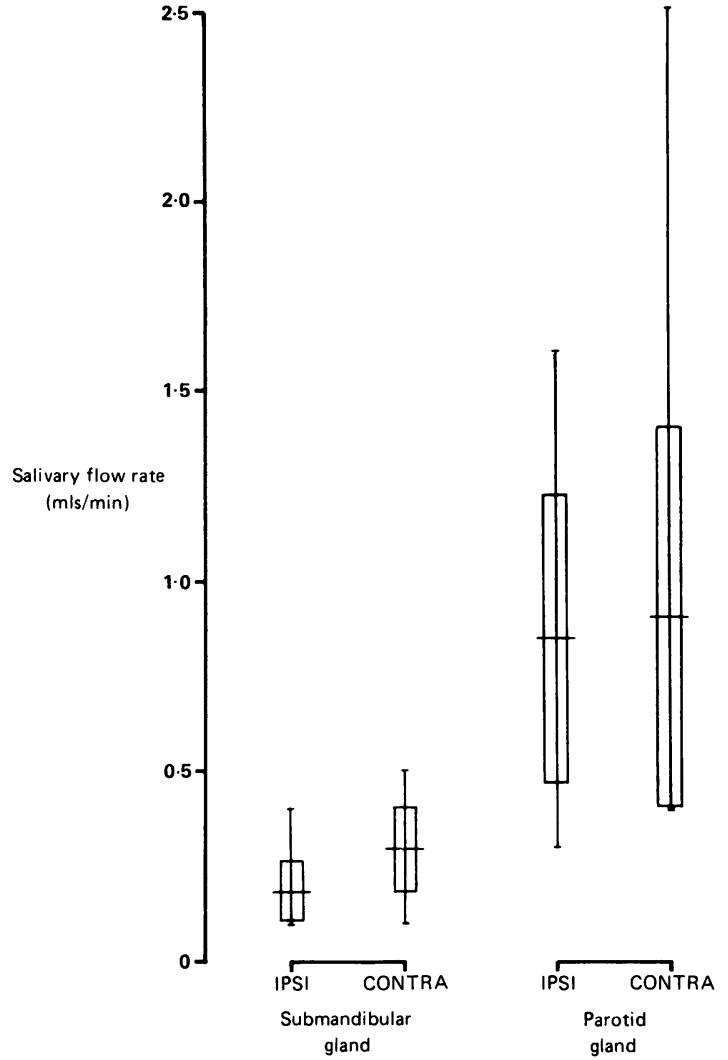

Figure Results of mean, $X 1$ standard deviation and range of ipsilateral and contralateral submandibular and parotid stimulated salivary flow rates.

On the affected side, the submandibular flow rate was less than that of the normal side in 13 patients, was the same in six patients and was greater than the normal side in one patient. There was a mean reduction in submandibular flow of $54 \%$ in the 13 patients with diminished flow. When the submandibular flow rate on the affected side and the healthy side were compared using paired parametric tests (one way analysis of variance) and non parametric tests (Kruskal Wallis One Way Analysis of Ranks) there was a highly significant difference between the sides $(p<0.0001)$. There was no statistically significant difference between the parotid salivary flow rates. There was not a statistically significant difference between the submandibular or parotid flow rates in the group that had salivary flow rates measured within eight weeks of operation (No. 2, 3, 4, 6, 9, 10, 11, 13, 16, 17, 19) and those measured after this time (No. 1, 5, 7, 8, 12, 14, 15, 18,20 ).

Symptomatically, only five patients were aware of any change in salivation post-operatively. Four of
Table 2 Individual results of electrogustometric taste detectable thresholds (EGTDT) and chemical taste recognition and detection thresholds following unilateral chorda tympani section

\begin{tabular}{|c|c|c|c|c|}
\hline \multirow[b]{3}{*}{$\begin{array}{l}\text { Subject } \\
\text { No. }\end{array}$} & \multicolumn{4}{|c|}{ Taste detection and recognition thresholds } \\
\hline & \multicolumn{2}{|c|}{ Ipsilateral } & \multicolumn{2}{|c|}{ Contralateral } \\
\hline & $\begin{array}{l}\text { EGTDT } \\
(\mu A)\end{array}$ & Chemical & $\begin{array}{l}\text { EGTDT } \\
(\mu A)\end{array}$ & Chemical \\
\hline 1 & 95 & $(1,2)$ & 3 & $(8,1)$ \\
\hline 2 & 327 & $(0,0)$ & 5 & $(10,2)$ \\
\hline 3 & 150 & $(0,0)$ & 2 & $(11,1)$ \\
\hline 4 & 647 & $(0,0)$ & 14 & $(9,3)$ \\
\hline 5 & 748 & $(0,0)$ & 6 & $(8,3)$ \\
\hline 6 & 414 & $(0,0)$ & 24 & $(11,0)$ \\
\hline 7 & 414 & $(0,0)$ & 22 & $(7,5)$ \\
\hline 8 & 114 & $(3,3)$ & 16 & $(11,1)$ \\
\hline 9 & 220 & $(0,0)$ & 17 & $(10,0)$ \\
\hline 10 & 156 & $(2,2)$ & 8 & $(9,3)$ \\
\hline 11 & 850 & $(0,0)$ & 90 & $(2,1)$ \\
\hline 12 & 313 & $(0,0)$ & 7 & $(11,1)$ \\
\hline 13 & 568 & $(0,0)$ & 15 & $(7,4)$ \\
\hline 14 & 501 & $(0,0)$ & 11 & $(4,7)$ \\
\hline 15 & 350 & $(0,1)$ & 9 & $(5,4)$ \\
\hline 16 & 433 & $(0,0)$ & 26 & $(6,5)$ \\
\hline 17 & 450 & $(4,2)$ & 6 & $(9,3)$ \\
\hline 18 & 120 & $(0,0)$ & 6 & $(7,2)$ \\
\hline 19 & 350 & $(1,4)$ & 16 & $(11,1)$ \\
\hline 20 & 700 & $(0,1)$ & 80 & $(5,5)$ \\
\hline
\end{tabular}

these patients (No. 5, 14, 15, 20), were aware of decreased salivation post-operatively which had settled completely by three weeks in three patients (No. 5 , $15,20)$. One patient felt that there had been an increase in salivary flow post-operatively (No. 17).

\section{Electrogustometric and chemical taste thresholds}

Prior to formal testing only two patients were aware of a decrease in taste recognition (No. 12, 20) following unilateral chorda tympani section and one patient (No. 17) complained of a metallic taste especially at night (dysgeusia).

The results of the taste detection and taste recognition thresholds, ranked for the patient's age, are shown in table 2.

Electrogustometric taste detection thresholds (EGTDT) were increased on the affected side in all cases ranging from at least twice the upper limit of normal (upper limit of normal $=40 \mu$ Amps) to 20 times the upper limit of normal. EGTDTs were also elevated on the un-operated "normal" side in two patients.

The results for 12 chemical taste solutions in each patient are presented in brackets as (No. correctly recognised, No. of tastes detected but not recognised). Therefore, 13 patients could not detect any of the 12 solutions on the operated side. Two patients could detect a taste from one of the 12 solutions but could not recognise it correctly (No. 15 and 20). The remaining five patients could recognise some of the 12 correctly and could detect some of the chemical tastes 
but not identify them. Of the latter five patients, none could correctly recognise more than $33 \%$ of taste solutions, that is, four of 12 (No. 17).

\section{Discussion}

Quoted values for daily human total salivary flow rate vary from $500 \mathrm{mls}$ to $1500 \mathrm{mls} .^{6}$ Some authors have found that both the resting and the stimulated submandibular salivary flow rates are greater than those of the parotid gland ${ }^{67}$ while others found that stimulated parotid and submandibular secretion were equal. ${ }^{8}$ Since we were interested in the effect of chorda tympani section, salivary flow was stimulated (using citric acid). This study demonstrated that stimulated salivary flow rate from the submandibular gland accounted for only $27 \%$ of the total salivary flow and that chorda tympani section only reduced submandibular salivary flow rate in $65 \%$ of cases.

In those patients who had a reduction in salivary flow, the mean reduction was by only $54 \%$. This was similar to success rates noted by other authors. ${ }^{9-12}$ It has been suggested that immediately after chorda tympani section, submandibular flow is at its lowest and that over the next few months the flow increases and that there may be "compensatory hypertrophy" in the contralateral gland.$^{13}$ However, these claims were based on only five patients, four of whom already had reduced submandibular salivary flow on the lesioned side pre-operatively; only three patients had a reduction in stimulated flow rate immediately postoperatively and two of the three had an increase in flow rate at 4.5 years post-operatively while one had a further decrease. The interpretation of the results of the contralateral submandibular flow were equally open to criticism.

Thomas $^{14}$ noted that salivary production was decreased by approximately $75 \%$ following chorda tympani section but others have reported significant secretory activity in the submandibular gland not only immediately after surgery but also after some months or years ${ }^{10-13}$ and suggest that the gland may be reinnervated. In our study, there did not appear to be any statistically significant difference when those tested less than eight weeks after operation were compared with those tested more than eight weeks post-operatively. There is also debate about the effect of chorda tympani section on ipsilateral parotid flow. Vollrath and others ${ }^{1516}$ studied the effect of chorda tympani section on ipsilateral parotid salivary flow and reported a reduction of almost $50 \%$. They concluded that the chorda tympani sends branches to the ipsilateral parotid gland. Vollrath et al's study ${ }^{15}$ could be criticised firstly, because their data were not paired and secondly because the parotid flow rate was $<1 \mathrm{ml}$ in $15 \mathrm{~min}$. on the ipsilateral side in nine patients and on the healthy contralateral side in four patients which would suggest that these patients either had parotid disease or that there had been technical difficulties in collecting saliva from the parotid glands. If the patients with flow rates $<1 \mathrm{ml}$ in $15 \mathrm{~min}$. were excluded, the range of values for parotid flow rate is similar in both sides and there does not appear to be any obvious difference between the groups. Nevertheless, parotid gland innervation from the chorda tympani has been pathologically confirmed in rabbits. ${ }^{17}$ Although the mean parotid flow of the group, in our patients, was less on the lesioned side this was not statistically significant.

Few patients are aware of any change in taste discrimination following unilateral chorda tympani section because taste is still perceived via the normal taste buds on the healthy side and the posterior one third of the tongue. Following bilateral chorda tympani section patients almost invariably are aware of loss of taste. All patients had elevated electrogustometric taste detection thresholds on the lesioned side and limited chemical taste recognition and detection. One patient (No. 11), with bilaterally elevated EGTDTs was a heavy smoker and had a "hairy" tongue, but there was no readily apparent cause for the elevated EGTDT on the "normal" side in the other patient (No. 20).

Chorda tympani section fails to reduce salivary flow in $35 \%$ of patients and in the remainder will only reduce submandibular salivary flow by approximately $54 \%$. We suggest that in patients with sialorrhoea and a limited life span, as in motor neuron disease, the morbidity of bilateral chorda tympani section outweighs the expected benefit and in these cases either alternative pharmacological methods of achieving control of drooling, chorda tympani section plus tympanic neurectomy or irradiation of the parotid or submandibular glands, should be considered.

\section{References}

1 Tormalin HG, Bjerre I. Surgical elimination of drooling. The Laryngoscope 1968;86:104-12.

2 Randell GM, Johnson KA, Patterson CN. Parasympathetic nerve section for control of sialorrhoea. Acta Otolaryngol 1977;103:94-7.

3 Frederick FJ, Stewart IF. Effectiveness of transtympanic neurectomy in management of sialorrhoea occurring in mentally retarded patients. J Otolaryngol 1982;11: 289-92.

4 Grant R, Ferguson MM, Strang R, Turner JW, Bone I. Evoked taste thresholds in normal population and the application of electrogustometry to trigeminal nerve disease. J Neurol Neurosurg Psychiatry 1987;50:12-21.

5 McKeown KC, Dunstone GH. Some observations on salivary secretion and fluid absorption by mouth. $\mathrm{Br}$ Med J 1959;ii:670-2. 
6 Ganong WF. Review of Medical Physiology, 10th ed. Los Altos: Lange Medical Publications, 1981;26:384-6.

7 Schneyer LH, Levin LE. Rate of salivary secretion. J Appl Physiol 1955;7:609-13.

8 Dawes $\mathrm{C}$, Wood CM. The contribution of oral minor mucous gland secretions to the volume of saliva in man. Arch Oral Biol 1973;18:337-42.

9 Towsend GL, Morimoto AM, Kralemann H. Management of sialorrhoea in mentally retarded patients by transtympanic neurectomy. Mayo Clinic Proc 1973;48:776-9.

10 Michel RG, Johnson KA, Patterson CN. Parasympathetic nerve section for the control of sialorrhoea. Arch Otolaryngol 1977;103:94-7.

11 Mullins WM, Gross CW, Moor JM. Longterm follow-up of tympanic neurectomy for sialorrhoea. Laryngoscope 1979;89:1219-23.
12 Arnold HG, Gross CW. Transtympanic neurectomycorrection of drooling in cerebral palsy. NY State $J$ Med 1975;75:2419-22.

13 Chilla R, Nicklatsch J, Arglebe C. Late sequlae of iatrogenic damage to chorda tympani nerve. Acta Otolaryngol 1982;94:461-5.

14 Thomas RL. Tympanic neurectomy and chorda tympani section. Aust N Z Surg 1980;50:352-5.

15 Vollrath M, Arglebe C, Chilla R. Die Bedeutung der chorda tympani fur die Parotisfunktion. Arch Otorhinolaryngol 1982;236:87-95.

16 Diamont $\mathrm{H}$, Wiberg A. Does the chorda tympani in man contain secretory fibres for the parotid gland? Acta Otolaryngol 1965;60:255-64.

17 Wallenborn WM. Parotid gland atrophy produced by transtympanic destruction of the tympanic plexus. Laryngoscope 1968;78:132-44. 